4 Clarke SC, Taffel S. Changes in cesarean delivery in the United States, 1988 and 1993. Birth 1995;22:63-67.

5 O’Driscoll K, Jackson RJA, Gallagher JT. Prevention of prolonged labour. BMJ 1969;2:477-480

6 O'Driscoll K, Foley M, MacDonald D. Active management of labor as an alternative to cesarean section for dystocia. Obstet Gynecol 1984;63:485-490.

7 Taffel SM, Placek PJ, Liss T. Trends in the United States cesarean section rate and reasons for the 1980-85 rise. Am J Public Health 1987;77:955-959.

8 Lopez-Zeno JA, Peaceman AM, Adashek JA, et al. A controlled trial of a program for the active management of labor. $N$ Engl J Med 1992;26:450-454
9 Frigoletto FD Jr, Lieberman E, Lang JM, et al. A clinical trial of active management of labor [erratum published in $N$ Engl J Med 1995;333:1163]. N Engl J Med 1995;333:745-750.

10 Rogers R, Gilson GJ, Miller AC, et al. Active management of labor: does it make a difference? Am J Obstet Gynecol 1997;177:599-605.

11 Gardner M. Cost analysis in obstetrics and gynecology. Clin Obstet Gynecol 1998;41:296-306.

12 Keeler EB. Decision analysis and cost-effectiveness analysis in women's health care. Clin Obstet Gynecol 1994;37:207-215.

13 Gardner M, Rouse DJ, Goldenberg RL, et al. Cost comparison of induction of labor versus expectant management in pregnancies lasting longer than 41 weeks. Am J Managed Care 1996;2:814-818.

\title{
COMMENTARY
}

\section{The active management of labor: is it worth the cost?}

In 1969, O'Driscoll and colleagues, in a seminal work published in the $B M J{ }^{1}$ detailed the active management of labor to prevent primigravid patients from laboring more than 24 hours. Not until 1992 was a similar activemanagement protocol shown in a randomized trial to shorten labor. ${ }^{2}$ Since that time, several randomized controlled trials have examined various components of active management and have shown reductions in the length of labor, with a trend toward fewer cesarean deliveries., ${ }^{3,4}$ None of the studies found an increase in adverse outcomes with this approach.

Given this evidence, why has active management (as described by O'Driscoll and coworkers) not become the standard of care in the United States? First, the original protocol calls for one-to-one support by nurses of women in labor, something not routinely offered in US hospitals. Second, O'Driscoll and associates describe as a fundamental mistake the current common practice of admitting patients before they are truly in labor-a useful way to ensure adequate pain control for a primigravid woman with painful contractions but no cervical change, but perhaps a substantial impediment to reducing the high cesarean delivery rate. Financial incentives may also be misaligned in the current US health care system. That is, attending obstetricians may have little interest in shortening labor and decreasing the use of cesarean sections if, as a result, they must work harder or get paid less for their time.

The cost analysis by Rogers and colleagues of the active management of labor attempts to show whether a thirdparty payer would have incentive to adopt such an approach. ${ }^{5}$ Cost analysis can play an important role in guiding the decisions of group health care providers, particularly decisions about two broad groups of interventions: those that improve health but increase cost, and those that worsen health but save money. ${ }^{6}$

Shortening labor and possibly reducing the rate of cesarean delivery rate would be considered by most to be improvements in health. Assuming that vaginal delivery is preferred over cesarean, and shorter labors are better than longer, then if active management saves money, or is neutral with regard to costs, it should be adopted. In general, we do not require interventions that improve health to save money. If an intervention costs money, then a costbenefit analysis would be needed.

The analysis by Rogers and associates depends on the former case being satisfied because they do not attempt to weigh the health consequences of active management. They conclude that cost savings derived from active management are minimal, if they exist at all. This finding is less well grounded than it first appears because of the way the authors handle a common problem in cost analysis. Specifically, faced with the difficult task of estimating costs, they substitute charges and sum the two. Their rationale - that they are assuming the position of a third-party payer-does not fully justify this decision. An indemnity insurance plan would be interested only in charges; that the hospital may charge more than it costs to deliver some services and less than it costs to deliver others would not matter to them one bit. A closed-panel health maintenance organization, on the other hand, would care only about costs; indeed, "charges" may be a meaningless term in that setting.

Summing costs and charges has the effect of muddying the waters with regard to the worth of active management. Consider what would happen if the cost of disposable equipment and charges for labor and delivery (two items for which the authors estimated cost) dropped dramatically in the active-management arm, but the hospital charge was unchanged (as would be expected if obstetric services are charged by diagnosis and procedure, not time). The large drop in cost would be swamped by the lack of change in charges. Even if such a drop produced a significant fall in overall cost, a third-party payer could hardly be faulted for not caring. After all, the cost savings have gone to the hospital's bottom line.
Michael S Broder Department of Obstetrics and Gynecology UCLA School of Medicine, Los Angeles RAND Health 1700 Main St PO Box 2138 Santa Monica, CA 90401-2138

Correspondence to: Dr Broder mbroder@rand.org Competing interests: None declared

West J Med 2000;172:243-244 
Even if charges reflected actual costs, other problems with adding costs and charges arise because charges may be two or three times the actual cost (accounting for, among other things, profit). For an item for which the authors estimated cost, a $\$ 1$ drop in cost from the control to the active-management arm would end up as a $\$ 1$ drop in the total. For an item measured by charges, a $\$ 1$ drop in cost might be reflected as a $\$ 2$ or $\$ 3$ drop in charges.

Evaluating cost alone, and doing it from society's perspective (rather than that of a third-party payer) would eliminate some of these problems. Charges can be converted to costs, although imperfectly, using cost-to-charges ratios available at most hospitals. Taking society's viewpoint also removes the problem of cost shifting inherent in this type of intervention. That is, if an intervention reduces hospital costs by shifting them to other parts of society (for example, discharging patients 48 hours after a cesarean section, thereby increasing care costs for patients' families), it looks good from the third-party perspective but bad from society's perspective.

Is active management worth the cost? Despite the flaws in their analysis, Rogers and colleagues make a strong argument that it is. Using charges and costs together is unlikely to hide a major increase in cost from this program. We do not usually demand that improvements in health come with cost savings, but rather that they do not cost too much. Providing shorter labors without complications at no cost is a pretty good deal. Further analysis might demonstrate cost savings, particularly if society's benefit from a reduced cesarean section rate is evaluated.

References

1 O'Driscoll K, Jackson RJA, Gallagher JT. Prevention of prolonged labour. BMJ 1969;2(655):477-480.

2 Lopez-Zeno JA, Peaceman AM, Adashek JA, et al. A controlled trial of a program for the active management of labor. $N$ Engl J Med 1992;326:450-454.

3 Frigoletto FD Jr, Lieberman E, Lang JM, et al. A clinical trial of active management of labor [erratum published in $N$ Engl J Med 1995;333:1163]. N Engl J Med 1995;333:745-750.

4 Rogers R, Gilson G, Miller AC, et al. Active management of labor: does it make a difference? Am J Obstet Gynecol 1997;177:599-605.

5 Rogers RG, Gardener MO, Tool KJ, et al. Active management of labor: a cost analysis of a randomized trial. West J Med 2000;172:240-243

6 Keeler EB. Decision analysis and cost-effectiveness analysis in women's health care. Clin Obstet Gynecol 1994;37:207-215.

\section{Note to our readers}

This month, for the first time, we recognize the contributions of our "hanging committee" on the wjm masthead. This group of knowledgeable and talented individuals volunteer a great deal of time and expertise to the journal. Experts in clinical epidemiology, statistics, and study design, they scrutinize all manuscripts previously subjected to peer review and found to merit serious consideration. They not only help decide on suitability for publication, but also provide methodologic advice and suggestions to prospective authors.

The "hanging committee" is not where manuscripts are sent to their execution. Rather, the term derives from an old British Medical Association custom (and one shared by many other privileged groups in the United Kingdom), where a special committee served as final arbiter of whether, and precisely where and how, a new portrait of some dignitary should be hung.

Whether and how to "hang" our submissions, in public, for the enjoyment and edification of our readers, is just about our most important job. We, therefore, are delighted to have this opportunity to thank this group of experts. We are lucky to have them. 